\title{
CRITERIA FOR UNIVALENCE AND QUASICONFORMAL EXTENSION FOR HARMONIC MAPPINGS ON PLANAR DOMAINS
}

\section{Iason Efraimidis}

Texas Tech University, Department of Mathematics and Statistics Box 41042, Lubbock, TX 79409, United States; iason.efraimidis@ttu.edu

\begin{abstract}
If $\Omega$ is a simply connected domain in $\overline{\mathbf{C}}$ then, according to the Ahlfors-Gehring theorem, $\Omega$ is a quasidisk if and only if there exists a sufficient condition for the univalence of holomorphic functions in $\Omega$ in relation to the growth of their Schwarzian derivative. We extend this theorem to harmonic mappings by proving a univalence criterion on quasidisks. We also show that the mappings satisfying this criterion admit a homeomorphic extension to $\overline{\mathbf{C}}$ and, under the additional assumption of quasiconformality in $\Omega$, they admit a quasiconformal extension to $\overline{\mathbf{C}}$. The Ahlfors-Gehring theorem has been extended to finitely connected domains $\Omega$ by Osgood, Beardon and Gehring, who showed that a Schwarzian criterion for univalence holds in $\Omega$ if and only if the components of $\partial \Omega$ are either points or quasicircles. We generalize this theorem to harmonic mappings.
\end{abstract}

\section{Introduction}

1.1. Schwarzian derivative. For a locally univalent analytic function $f$ the Schwarzian derivative is defined by

$$
S f=\left(f^{\prime \prime} / f^{\prime}\right)^{\prime}-\frac{1}{2}\left(f^{\prime \prime} / f^{\prime}\right)^{2} .
$$

This operator vanishes identically if and only if $f$ is a Möbius transformation. According to a classical theorem of Nehari the bound

$$
|S f(z)| \leq \frac{2 t}{\left(1-|z|^{2}\right)^{2}}, \quad z \in \mathbf{D},
$$

for $t=1$ implies the global univalence of $f$ in the unit disk $\mathbf{D}$, while another classical result, proved by Ahlfors and Weill, gives an explicit quasiconformal extension of $f$ to $\overline{\mathbf{C}}$ under the assumption that $f$ satisfies (1) with $t<1$.

Let $\Omega$ be a hyperbolic domain in $\overline{\mathbf{C}}$, meaning that it has at least three boundary points, and let $\pi: \mathbf{D} \rightarrow \Omega$ be a universal covering map. Then the density $\lambda_{\Omega}$ of the hyperbolic (Poincaré) metric in $\Omega$ is defined by

$$
\lambda_{\Omega}(\pi(z))\left|\pi^{\prime}(z)\right|=\lambda_{\mathbf{D}}(z)=\frac{1}{1-|z|^{2}}, \quad z \in \mathbf{D},
$$

and is independent of the choice for the covering $\pi$. The size of the Schwarzian derivative of a locally univalent holomorphic function $f: \Omega \rightarrow \mathbf{C}$ is measured by its norm, given by

$$
\|S f\|_{\Omega}=\sup _{z \in \Omega} \lambda_{\Omega}(z)^{-2}|S f(z)|
$$

https://doi.org/10.5186/aasfm.2021.4669

2020 Mathematics Subject Classification: Primary 30C55, 30C62, 31A05.

Key words: Harmonic mappings, Schwarzian derivative, univalence criterion, quasiconformal extension. 
The inner radius of $\Omega$ is defined as the number

$$
\sigma(\Omega)=\sup \left\{c \geq 0:\|S f\|_{\Omega} \leq c \Rightarrow f \text { univalent }\right\} .
$$

This domain constant is Möbius invariant. We say that a univalence criterion holds in $\Omega$ if and only if $\sigma(\Omega)>0$. We have that $\sigma(\mathbf{D})=2$ since, as shown by Hille, the constant 2 in Nehari's theorem is sharp. Lehtinen showed that every simply connected domain $\Omega$ satisfies $\sigma(\Omega) \leq 2$, with equality only in the case when $\Omega$ is a disk or a half-plane. See Lehto's book [14, Ch. III, §5] for more information on the inner radius.

A domain $\Omega$ is a quasidisk if it is the image of $\mathbf{D}$ under a quasiconformal self-map of $\overline{\mathbf{C}}$. The boundary of a quasidisk is called a quasicircle.

Our starting point is the following theorem by Ahlfors and Gehring.

Theorem A. $[1,7]$ Let $\Omega$ be a simply connected domain in $\overline{\mathbf{C}}$. Then $\sigma(\Omega)>0$ if and only if $\Omega$ is a quasidisk. Moreover, any holomorphic function $f: \Omega \rightarrow \mathbf{C}$ that satisfies $\|S f\|_{\Omega}<\sigma(\Omega)$ admits a quasiconformal extension to $\overline{\mathbf{C}}$.

Ahlfors [1] proved the quasiconformal extension criterion on a quasidisk stated here; the univalence criterion follows from it. The fact that such a criterion can only occur on a quasidisk was shown by Gehring [7]. This has been generalized to a class of multiply connected domains with the following theorem.

Theorem B. $[2,18]$ Let $\Omega$ be a finitely connected domain in $\overline{\mathbf{C}}$. Then $\sigma(\Omega)>0$ if and only if every boundary component of $\Omega$ is either a point or a quasicircle.

The univalence criterion was proved by Osgood [18], while the fact that the class of domains where it holds cannot be enlarged was proved by Beardon and Gehring [2]. We note that for finitely connected domains the property of $\partial \Omega$ described in this theorem characterizes the class of uniform domains, introduced by Martio and Sarvas [16] (see also [8, §3.5]). We also mention that there exist both examples of infinitely connected circle domains with positive (see [18]) and zero (see [2]) inner radius.

In recent years there is much activity in extending the theory of the Schwarzian derivative to harmonic mappings, to which we now turn.

1.2. Harmonic mappings. A complex-valued harmonic mapping $f$ in a simply connected domain $\Omega \subset \mathbf{C}$ has a canonical decomposition $f=h+\bar{g}$, where $h$ and $g$ are analytic in $\Omega$. The mapping $f$ is locally univalent if and only if its Jacobian $J_{f}=$ $\left|h^{\prime}\right|^{2}-\left|g^{\prime}\right|^{2}$ does not vanish, and is said to be orientation-preserving if its dilatation $\omega=g^{\prime} / h^{\prime}$ satisfies $|\omega|<1$ in $\Omega$. We say that $f$ is normalized if $h\left(z_{0}\right)=g\left(z_{0}\right)=0$ and $h^{\prime}\left(z_{0}\right)=1$ for some specified $z_{0} \in \Omega$.

The Schwarzian derivative has been extended to harmonic mappings by two complementary definitions: a first one appeared in [4] and another was later introduced by Hernández and Martín in [11]. We will follow the latter, which seems to be better suited when one does not wish to consider the Weierstarss-Enneper lift to a minimal surface. Hence, the Schwarzian derivative of a locally univalent harmonic mapping $f$ is defined by

$$
S_{f}=\rho_{z z}-\frac{1}{2}\left(\rho_{z}\right)^{2}, \quad \rho=\log J_{f},
$$

where $J_{f}=\left|f_{z}\right|^{2}-\left|f_{\bar{z}}\right|^{2}$ is the Jacobian. If $\Omega$ is simply connected and, therefore, the decomposition $f=h+\bar{g}$ is valid, then the above takes the form

$$
S_{f}=S h+\frac{\bar{\omega}}{1-|\omega|^{2}}\left(\frac{h^{\prime \prime}}{h^{\prime}} \omega^{\prime}-\omega^{\prime \prime}\right)-\frac{3}{2}\left(\frac{\omega^{\prime} \bar{\omega}}{1-|\omega|^{2}}\right)^{2} .
$$


Note that we are using the notation $S f$ when we know that the mapping $f$ is holomorphic and the notation $S_{f}$, with the mapping $f$ as a subscript, in the more general setting of harmonic mappings. This operator satisfies the chain rule, for if $\varphi$ is analytic in $\Omega$ and such that the composition $f \circ \varphi$ is well defined then we have that

$$
S_{f \circ \varphi}=S_{f} \circ \varphi\left(\varphi^{\prime}\right)^{2}+S \varphi .
$$

The Schwarzian norm of a harmonic mapping $f$ is defined exactly as in (2). It was shown in [11] that $\left\|S_{f}\right\|_{\Omega}=0$ implies that $f$ is an affine map of a Möbius transformation and is, therefore, univalent.

1.3. Main results. We define the harmonic inner radius of a hyperbolic domain $\Omega$ in $\overline{\mathbf{C}}$ as the constant

$$
\sigma_{H}(\Omega)=\sup \left\{t \geq 0: f \text { harmonic in } \Omega \text { with }\left\|S_{f}\right\|_{\Omega} \leq t \Rightarrow f \text { univalent }\right\} .
$$

Evidently,

$$
\sigma_{H}(\Omega) \leq \sigma(\Omega)
$$

since every holomorphic function is harmonic. This shows that if $\sigma_{H}(\Omega)>0$ and $\Omega$ is finitely connected then, in view of Theorem $\mathrm{B}$, every boundary component of $\Omega$ is either a point or a quasicircle. For $\Omega=\mathbf{D}$ it was shown in [12] that $\sigma_{H}(\mathbf{D})>0$. We prove that the harmonic inner radius is positive for all quasidisks.

Theorem 1. Let $\Omega$ be a quasidisk. Then there exists a constant $c>0$, depending only on $\sigma(\Omega)$, such that if $f$ is harmonic in $\Omega$ with $\left\|S_{f}\right\|_{\Omega} \leq c$ then $f$ is univalent in $\Omega$ and admits a homeomorphic extension to $\overline{\mathbf{C}}$.

The proof of the univalence criterion in Theorem 1 follows closely the reasoning in [12]: We show that if a harmonic mapping $f=h+\bar{g}$ has small Schwarzian derivative then so does its analytic part $h$, and therefore $h$ is univalent by Theorem A. The same can then be said about $h+a g$, the analytic part of the affine transformation $f+a \bar{f}$, $a \in \mathbf{D}$. Finally, Hurwitz' theorem shows that $h+a g$ is univalent for every $a \in \overline{\mathbf{D}}$ and by an elementary rotational argument we get that $f$ is injective. The crucial step in the generalization from $\mathbf{D}$ to a quasidisk involves the hyperbolic derivative of admissible dilatations and is given in Lemma 5. The homeomorphic extension under these hypotheses is novel even for the unit disk.

Further, for more general domains we prove the following theorem.

Theorem 2. Let $\Omega$ be a finitely connected domain. The following are equivalent.

(i) Every boundary component of $\Omega$ is either a point or a quasicircle.

(ii) $\sigma(\Omega)>0$.

(iii) $\sigma_{H}(\Omega)>0$.

The equivalence of (i) and (ii) was given in Theorem B, while the direction (iii) $\Rightarrow$ (ii) follows trivially from (5). We prove the direction (i) $\Rightarrow$ (iii) in Section 6 by using Osgood's [18] quasiconformal decomposition of $\Omega$ and the homeomorphic extension of Theorem 1.

Finally, we give sufficient conditions for a harmonic mapping defined on a quasidisk to admit a quasiconformal extension to $\overline{\mathbf{C}}$.

Theorem 3. Let $\Omega$ be a quasidisk and let $d \in[0,1)$. Then there exists a constant $c>0$, depending only on $d$ and on $\sigma(\Omega)$, such that if $f$ is harmonic in $\Omega$ with $\left\|S_{f}\right\|_{\Omega} \leq c$ and its dilatation satisfies $\sup _{z \in \Omega}|\omega(z)| \leq d$ then $f$ admits a quasiconformal extension to $\overline{\mathbf{C}}$. 
If $d=0$ then $f$ is analytic and we recover Ahlfors' theorem [1]. For our proof of Theorem 3 we consider the dilation $\Omega_{r}$ (i.e. the image of $|z|<r$, for $r<1$, under the Riemman mapping of $\Omega$ ) and use quasiconformal reflections to obtain a $K$-quasiconformal extension of $\left.f\right|_{\Omega_{r}}$ to $\overline{\mathbf{C}}$. The desired extension is then harvested as the limit for $r \rightarrow 1$, once we prove that $K$ is independent of $r$ by studying the cross-ratio of points on the image of $\partial \Omega_{r}$ under $f$. For the latter we use the insightful ideas of [10]. We prove Theorem 3 in Section 5.

We note that for the case when $\Omega$ is the unit disk $\mathbf{D}$, Theorem 3 was proved in [12]. However, the slightly stronger statement made there, namely that the constants $c$ and $d$ in the hypotheses are independent, does not seem to follow from the suggestion of the authors to argue as in [10] for a proof. It is interesting to ask if this stronger statement can be rigorously proved.

\section{Preliminaries}

2.1. Bounded Schwarzian derivative. A well-known theorem of Pommerenke [19] states that if $\varphi$ is analytic and locally univalent in $\mathbf{D}$ then

$$
\left(1-|z|^{2}\right)\left|\frac{\varphi^{\prime \prime}(z)}{\varphi^{\prime}(z)}-\frac{2 \bar{z}}{1-|z|^{2}}\right| \leq 2 \sqrt{1+\frac{\|S \varphi\|_{\mathbf{D}}}{2}}, \quad z \in \mathbf{D} .
$$

From this, a simple application of Montel's theorem shows that the set of functions

$$
\left\{\varphi:\|S \varphi\|_{\mathbf{D}} \leq c\right\},
$$

where $c>0$, constitutes a normal family.

According to Theorem 6 in [11], if $f=h+\bar{g}$ is harmonic and locally univalent in $\mathbf{D}$ then

$$
\left\|S_{f}\right\|_{\mathbf{D}}<\infty \text { if and only if }\|S h\|_{\mathbf{D}}<\infty .
$$

2.2. Normalizations for harmonic mappings. Let $\Omega$ be a simply connected domain that contains the origin and let $t \geq 0$. We then denote by $\mathcal{F}_{t}(\Omega)$ the set of all sense-preserving harmonic mappings $f=h+\bar{g}$ in $\Omega$ which satisfy $\left\|S_{f}\right\|_{\Omega} \leq t$ and are normalized by $h(0)=g(0)=0$ and $h^{\prime}(0)=1$. Let

$$
\mathcal{F}_{t}^{0}(\Omega)=\left\{f \in \mathcal{F}_{t}(\Omega): g^{\prime}(0)=0\right\} .
$$

The following proposition is well known among experts but we will include a proof here for the convenience of the reader. We note that the compactness of $\mathcal{F}_{t}^{0}(\mathbf{D})$ was shown in [5].

Proposition 1. The family $\mathcal{F}_{t}(\Omega)$ is normal. The family $\mathcal{F}_{t}^{0}(\Omega)$ is normal and compact.

Proof. To show that $\mathcal{F}_{t}^{0}(\mathbf{D})$ is normal we observe that the set

$$
\left\{h: f=h+\bar{g} \in \mathcal{F}_{t}^{0}(\mathbf{D})\right\}
$$

is a normal family in view of inequality (6) and since $\|S h\|_{\mathbf{D}}$ is bounded by (7) (even more so, a close inspection of [11, Thm. 6] shows that it is uniformly bounded). Also, again by (6), the functions $h^{\prime}$ are locally uniformly bounded in $\mathbf{D}$ and, in view of the condition $\left|g^{\prime}\right|<\left|h^{\prime}\right|$, so are the functions $g^{\prime}$. Hence the family $\left\{g: f \in \mathcal{F}_{t}^{0}(\mathbf{D})\right\}$ is also normal. Thus $\mathcal{F}_{t}^{0}(\mathbf{D})$ is a normal family.

For a simply connected domain $\Omega$, with $0 \in \Omega$, let $f \in \mathcal{F}_{t}^{0}(\Omega)$ and consider the mapping $F=\varphi^{\prime}(0)^{-1} f \circ \varphi$, where $\varphi$ is a Riemann map for which $\Omega=\varphi(\mathbf{D})$ and 
$\varphi(0)=0$. By the chain rule (4) we have that

$$
S_{F}(z)=S_{f}(\varphi(z)) \varphi^{\prime}(z)^{2}+S \varphi(z), \quad z \in \mathbf{D} .
$$

We then compute

$$
\frac{\left|S_{F}(z)-S \varphi(z)\right|}{\lambda_{\mathbf{D}}(z)^{2}}=\frac{\left|S_{f}(\varphi(z))\right|}{\lambda_{\Omega}(\varphi(z))^{2}}, \quad z \in \mathbf{D},
$$

which shows that $\left\|S_{F}-S \varphi\right\|_{\mathbf{D}}=\left\|S_{f}\right\|_{\Omega}$. According to Kraus' theorem [14, Ch. II, Thm. 1.3] we have that $\|S \varphi\|_{\mathbf{D}} \leq 6$. Therefore, we get that

$$
\left\|S_{F}\right\|_{\mathbf{D}} \leq\left\|S_{f}\right\|_{\Omega}+\|S \varphi\|_{\mathbf{D}} \leq t+6
$$

Hence the set $\left\{\varphi^{\prime}(0)^{-1} f \circ \varphi: f \in \mathcal{F}_{t}^{0}(\Omega)\right\}$ is included in the normal family $\mathcal{F}_{t+6}^{0}(\mathbf{D})$ and is, therefore, a normal family itself. The claim that $\mathcal{F}_{t}^{0}(\Omega)$ is normal follows directly from this.

Any $f \in \mathcal{F}_{t}(\Omega)$ can be written as an affine transform of a mapping in $\mathcal{F}_{t}^{0}(\Omega)$, in particular, if $b_{1}=g^{\prime}(0)$ we may write $f=f_{0}+\overline{b_{1}} \overline{f_{0}}$ for some $f_{0} \in \mathcal{F}_{t}^{0}(\Omega)$. Hence $|f| \leq 2\left|f_{0}\right|$ and with the observation that Montel's criterion for normality remains valid for families of harmonic mappings (see $\left[6\right.$, p.80]) we conclude that $\mathcal{F}_{t}(\Omega)$ is normal.

Finally, the compactness of $\mathcal{F}_{t}^{0}(\Omega)$, as shown in the course of the proof of Theorem 3 in [5], amounts to the observation that if $f_{n}$ is a sequence in $\mathcal{F}_{t}^{0}(\Omega)$ that converges to $f$ locally uniformly in $\Omega$ then $S_{f_{n}} \rightarrow S_{f}$ pointwise in $\Omega$. Hence $f \in \mathcal{F}_{t}^{0}(\Omega)$ and so this class is compact.

2.3. Affine invariance. Let $a \in \mathbf{D}$ and consider the affine transformation of a mapping $f$ in $\mathcal{F}_{t}(\Omega)$ given by

$$
F(z)=A_{a} f(z)=\frac{f(z)+a \overline{f(z)}}{1+a g^{\prime}(0)}, \quad z \in \Omega .
$$

Now $F \in \mathcal{F}_{t}(\Omega)$ since it satisfies $S_{F} \equiv S_{f}$ (see [11, Prop. 1]) and the corresponding normalizations. It can easily be seen that the dilatation of $F$ is given by $\omega_{F}=\nu \varphi_{\bar{a}} \circ \omega$, where $\nu \in \mathbf{T}(=\partial \mathbf{D})$ and

$$
\varphi_{a}(z)=\frac{a+z}{1+\bar{a} z}, \quad z \in \mathbf{D} .
$$

If we make the choice $a=-\overline{\omega(0)}$ then $F$ will have the additional normalization $\omega_{F}(0)=\nu \varphi_{\bar{a}}(-\bar{a})=0$ and will therefore belong to $\mathcal{F}_{t}^{0}(\Omega)$.

2.4. Quasiconformal mappings. A sense-preserving homeomorphism $f: \Omega \rightarrow$ C is said to be $K$-quasiconformal, $K \geq 1$, if it is absolutely continuous on lines and satisfies $\left|f_{\bar{z}}\right| \leq k\left|f_{z}\right|$, where $k=(K-1) /(K+1)$, almost everywhere in $\Omega$. A mapping is called quasiconformal if it is $K$-quasiconformal for some $K \geq 1$. The 1-quasiconformal mappings are the conformal mappings. Note that a harmonic mapping is quasiconformal if its dilatation satisfies $|\omega| \leq k<1$.

A domain $\Omega$ is called a $K$-quasidisk, and its boundary a $K$-quasicircle, if it is the image of $\mathbf{D}$ under a $K$-quasiconformal self-map of $\overline{\mathbf{C}}$. According to a theorem of Ahlfors [1] a Jordan curve $\gamma \subset \overline{\mathbf{C}}$ is a quasicircle if and only if for all points $z_{j} \in \gamma, j=1,2,3,4$, such that $z_{1}$ and $z_{3}$ separate $z_{2}$ and $z_{4}$, the cross-ratio

$$
\left(z_{1}, z_{2}, z_{3}, z_{4}\right)=\frac{\left(z_{1}-z_{2}\right)\left(z_{3}-z_{4}\right)}{\left(z_{1}-z_{3}\right)\left(z_{2}-z_{4}\right)}
$$


satisfies

for some constant $C>0$.

$$
\left|\left(z_{1}, z_{2}, z_{3}, z_{4}\right)\right| \leq C
$$

Let $\Omega_{1}$ and $\Omega_{2}$ be the complementary components of a Jordan curve $\gamma \subset \overline{\mathbf{C}}$. Then a sense-reversing homeomorphism $\lambda$ of the sphere onto itself is a reflection across $\gamma$ if it maps $\Omega_{1}$ onto $\Omega_{2}$ and keeps every point on $\gamma$ fixed. According to a theorem of Kühnau [13] (see also Theorem 2.1.4 in [8]) $\gamma$ is a $K$-quasicircle if and only if it admits a reflection $\lambda$ such that $\lambda(\bar{z})$ is $K$-quasiconformal.

\section{Preparatory lemmas}

3.1. Adaptations to general domains. The following proposition is a straightforward generalization of (7).

Proposition 2. Let $\Omega$ be a simply connected domain and $f=h+\bar{g}$ a sensepreserving locally univalent harmonic mapping in $\Omega$. Then $\left\|S_{f}\right\|_{\Omega}<\infty$ if and only if $\left\|S_{h}\right\|_{\Omega}<\infty$.

Proof. Let $\varphi$ be a Riemann map for which $\Omega=\varphi(\mathbf{D})$ and consider the mappings $F=f \circ \varphi$ and $H=h \circ \varphi$. By a calculation we saw in (8) we readily have that

$$
\left\|S_{F}-S \varphi\right\|_{\mathbf{D}}=\left\|S_{f}\right\|_{\Omega} \text { and }\|S H-S \varphi\|_{\mathbf{D}}=\|S h\|_{\Omega} .
$$

The proposition is a direct consequence of Theorem 6 in [11] and Kraus' theorem $\|S \varphi\|_{\mathbf{D}} \leq 6$.

We now generalize inequality (6) to an arbitrary hyperbolic domain $\Omega$. We write $d(z)=\operatorname{dist}(z, \partial \Omega)$ for the distance of a point $z$ in $\Omega$ to the boundary $\partial \Omega$.

Proposition 3. If $h$ is analytic and locally univalent in $\Omega$ then

$$
\left|\frac{h^{\prime \prime}(z)}{h^{\prime}(z)}\right| \leq \frac{2}{d(z)} \sqrt{1+\frac{\|S h\|_{\Omega}}{2}}, \quad z \in \Omega .
$$

Proof. Let $\alpha \in \Omega, d=d(\alpha)$ and consider the disk $\Delta=\{z:|z-\alpha|<d\}$. We write $z=\alpha+d \zeta \in \Delta$, for $\zeta \in \mathbf{D}$, and note that $d \lambda_{\Delta}(z)=\lambda_{\mathbf{D}}(\zeta)$. Since $\Delta \subset \Omega$ we have by the comparison principle [3, Thm. 8.1] that $\lambda_{\Delta}(z) \geq \lambda_{\Omega}(z)$, for all $z \in \Delta$. Let $H(\zeta)=h(z)$ and observe that $S H(\zeta)=d^{2} S h(z)$. We have that

$$
\|S H\|_{\mathbf{D}}=\sup _{\zeta \in \mathbf{D}} \frac{|S H(\zeta)|}{\lambda_{\mathbf{D}}(\zeta)^{2}}=\sup _{z \in \Delta} \frac{|S h(z)|}{\lambda_{\Delta}(z)^{2}} \leq \sup _{z \in \Omega} \frac{|S h(z)|}{\lambda_{\Omega}(z)^{2}}=\|S h\|_{\Omega} .
$$

We now apply inequality (6) to the function $H$ and evaluate at $\zeta=0$ to obtain

$$
d(\alpha)\left|\frac{h^{\prime \prime}(\alpha)}{h^{\prime}(\alpha)}\right|=\left|\frac{H^{\prime \prime}(0)}{H^{\prime}(0)}\right| \leq 2 \sqrt{1+\frac{\|S H\|_{\mathbf{D}}}{2}} \leq 2 \sqrt{1+\frac{\|S h\|_{\Omega}}{2}} .
$$

The proof is complete.

3.2. The hyperbolic derivative. If $\omega: \Omega \rightarrow \mathbf{D}$ is an analytic function then its hyperbolic derivative is given by

$$
\omega^{*}(z)=\frac{\omega^{\prime}(z)}{\lambda_{\Omega}(z)\left(1-|\omega(z)|^{2}\right)}, \quad z \in \Omega
$$

and the quantity $\left\|\omega^{*}\right\|=\sup _{z \in \Omega}\left|\omega^{*}(z)\right|$ is called the hyperbolic norm of $\omega$. In view of the generalized Schwarz-Pick lemma [3, Thm. 10.5] we always have that $\left\|\omega^{*}\right\| \leq 1$. The hyperbolic derivative satisfies the chain rule $(\omega \circ \varphi)^{*}=\omega^{*} \circ \varphi \cdot \varphi^{*}$ for any two functions for which the composition is well defined. 
It has been shown in [9] that for any analytic $\omega: \mathbf{D} \rightarrow \mathbf{D}$ it holds that

$$
\frac{\left(1-|z|^{2}\right)^{2}\left|\omega^{\prime \prime}(z)\right|}{1-|\omega(z)|^{2}} \leq C\left\|\omega^{*}\right\|, \quad z \in \mathbf{D}
$$

for some constant $C>0$. We will now generalize this to an arbitrary hyperbolic domain $\Omega$. Again, here $d(z)=\operatorname{dist}(z, \partial \Omega)$.

Proposition 4. If $\omega: \Omega \rightarrow \mathbf{D}$ is analytic then

$$
\frac{d(z)^{2}\left|\omega^{\prime \prime}(z)\right|}{1-|\omega(z)|^{2}} \leq C\left\|\omega^{*}\right\|, \quad z \in \Omega,
$$

for some constant $C>0$.

Proof. We proceed as in the proof of Proposition 3, by fixing $\alpha \in \Omega$ and writing $d=d(\alpha), \Delta=\{z:|z-\alpha|<d\}$ and $z=\alpha+d \zeta \in \Delta$, for $\zeta \in \mathbf{D}$. We set $\psi(\zeta)=\omega(z)$ and compute

$$
\begin{aligned}
\left\|\psi^{*}\right\| & =\sup _{\zeta \in \mathbf{D}} \frac{\left|\psi^{\prime}(\zeta)\right|}{\lambda_{\mathbf{D}}(\zeta)\left(1-|\psi(\zeta)|^{2}\right)}=\sup _{z \in \Delta} \frac{\left|\omega^{\prime}(z)\right|}{\lambda_{\Delta}(z)\left(1-|\omega(z)|^{2}\right)} \\
& \leq \sup _{z \in \Omega} \frac{\left|\omega^{\prime}(z)\right|}{\lambda_{\Omega}(z)\left(1-|\omega(z)|^{2}\right)}=\left\|\omega^{*}\right\| .
\end{aligned}
$$

The proof is completed by applying inequality (10) for $\zeta=0$ to the function $\psi$.

3.3. Admissible dilatations. Let $\Omega$ be a simply connected domain with $0 \in \Omega$ and let $\mathcal{A}_{t}(\Omega)$ and $\mathcal{A}_{t}^{0}(\Omega)$ denote the classes of admissible dilatations for mappings in $\mathcal{F}_{t}(\Omega)$ and $\mathcal{F}_{t}^{0}(\Omega)$, respectively. Let also

$$
R_{t}(\Omega)=\max _{\omega \in \mathcal{A}_{t}^{0}(\Omega)}\left\|\omega^{*}\right\|
$$

Applying the affine transformation (9) to a mapping $f \in \mathcal{F}_{t}(\Omega)$, as we have already seen, we can get a mapping $F=A_{a} f$ which, for an appropriate choice of $a \in \mathbf{D}$, belongs to $\in \mathcal{F}_{t}^{0}(\Omega)$. The dilatation of $F$ is $\omega_{F}=\nu \varphi_{\bar{a}} \circ \omega$, for some $\nu \in \mathbf{T}$. A straightforward computation can show that $\left|\omega_{F}^{*}\right|=\left|\omega^{*}\right|$, so that we have an alternative expression for $R_{t}$ given by

$$
R_{t}(\Omega)=\max _{\omega \in \mathcal{A}_{t}(\Omega)}\left\|\omega^{*}\right\|
$$

This was first observed in [5, Lem. 1]. A compactness argument was used in [12] to show that $R_{t}(\mathbf{D}) \rightarrow 0$ as $t \rightarrow 0^{+}$. Here we will prove the following.

Lemma 5. It holds that $R_{t}(\Omega) \leq 4 R_{t}(\mathbf{D})$ for all $t>0$.

An immediate consequence is that $R_{t}(\Omega) \rightarrow 0$ as $t \rightarrow 0^{+}$, and this fact is an important ingredient in the proofs of Theorems 1 and 3 . For the proof of Lemma 5 we need to recall the inequalities

$$
\frac{1}{4} \leq d(z) \lambda_{\Omega}(z) \leq 1, \quad z \in \Omega,
$$

where $d(z)=\operatorname{dist}(z, \partial \Omega)$, which amount to Koebe's 1/4-theorem and the comparison principle; see [14, Ch. I, §1.1].

Proof of Lemma 5. Fix $t>0$ and consider an extremal mapping $f=h+\bar{g}$ in $\mathcal{F}_{t}^{0}(\Omega)$, with dilatation $\omega$, for which $R_{t}(\Omega)=\left\|\omega^{*}\right\|$. There exists a sequence of points $\left\{z_{n}\right\}$ in $\Omega$ for which $\left\|\omega^{*}\right\|=\lim _{n \rightarrow \infty}\left|\omega^{*}\left(z_{n}\right)\right|$. Let $r_{n}=d\left(z_{n}\right)$ and consider the disks $\Delta_{n}=\left\{z:\left|z-z_{n}\right|<r_{n}\right\}$. Hereafter we use the notation $\zeta \in \mathbf{D}$ and $z=z_{n}+r_{n} \zeta \in \Delta_{n}$. 
Note that $r_{n} \lambda_{\Delta_{n}}(z)=\lambda_{\mathbf{D}}(\zeta)$ and that $\lambda_{\Delta_{n}}(z) \geq \lambda_{\Omega}(z)$, for $z \in \Delta_{n}$, since $\Delta_{n} \subset \Omega$. Let

$$
F_{n}(\zeta)=\frac{f(z)-f\left(z_{n}\right)}{r_{n} h^{\prime}\left(z_{n}\right)}
$$

and compute its dilatation $\omega_{n}(\zeta)=\mu_{n} \omega(z)$, where $\mu_{n}=h^{\prime}\left(z_{n}\right) / \overline{h^{\prime}\left(z_{n}\right)} \in \mathbf{T}$. Compute also $S_{F_{n}}(\zeta)=r_{n}^{2} S_{f}(z)$. We have that

$$
\frac{\left|S_{F_{n}}(\zeta)\right|}{\lambda_{\mathbf{D}}(\zeta)^{2}}=\frac{\left|S_{f}(z)\right|}{\lambda_{\Delta_{n}}(z)^{2}} \leq \frac{\left|S_{f}(z)\right|}{\lambda_{\Omega}(z)^{2}}
$$

so that

$$
\left\|S_{F_{n}}\right\|_{\mathbf{D}} \leq\left\|S_{f}\right\|_{\Omega} \leq t
$$

and, therefore, we find that $F_{n}$ belongs to $\mathcal{F}_{t}(\mathbf{D})$. Recalling the expression (11) we have that $\left\|\omega_{n}^{*}\right\| \leq R_{t}(\mathbf{D})$. A calculation shows that

$$
\omega_{n}^{*}(\zeta)=\frac{\omega_{n}^{\prime}(\zeta)}{\lambda_{\mathbf{D}}(\zeta)\left(1-\left|\omega_{n}(\zeta)\right|^{2}\right)}=\frac{\mu_{n} \omega^{\prime}(z)}{\lambda_{\Delta_{n}}(z)\left(1-|\omega(z)|^{2}\right)}=\mu_{n} \frac{\lambda_{\Omega}(z)}{\lambda_{\Delta_{n}}(z)} \omega^{*}(z)
$$

and, in particular, that

$$
\left|\omega_{n}^{*}(0)\right|=r_{n} \lambda_{\Omega}\left(z_{n}\right)\left|\omega^{*}\left(z_{n}\right)\right| .
$$

In view of Koebe's $1 / 4$-theorem (12) we have that $r_{n} \lambda_{\Omega}\left(z_{n}\right) \geq 1 / 4$. Therefore,

$$
\left|\omega^{*}\left(z_{n}\right)\right| \leq 4\left|\omega_{n}^{*}(0)\right| \leq 4\left\|\omega_{n}^{*}\right\| \leq 4 R_{t}(\mathbf{D}) \text {. }
$$

The proof is completed upon letting $n \rightarrow \infty$.

\section{Proof of Theorem 1}

Without loss of generality we may assume that $0 \in \Omega$ and that $f \in \mathcal{F}_{t}(\Omega), t \geq 0$. By Proposition 2 we have that $\|S h\|_{\Omega}<\infty$. Moreover, since the classes $\mathcal{F}_{t}(\Omega)$ are nested and increasing with $t$ we have that $\|S h\|_{\Omega}$ is uniformly bounded for $f \in \mathcal{F}_{t}(\Omega)$ and small $t$, that is, $\|S h\|_{\Omega} \leq M$ for some constant $M>0$ and, say, $t \leq 1$. From the expression (3) we get

$$
|S h| \leq\left|S_{f}\right|+\frac{\left|\omega^{\prime}\right|}{1-|\omega|^{2}}\left|\frac{h^{\prime \prime}}{h^{\prime}}\right|+\frac{\left|\omega^{\prime \prime}\right|}{1-|\omega|^{2}}+\frac{3}{2}\left(\frac{\left|\omega^{\prime}\right|}{1-|\omega|^{2}}\right)^{2} .
$$

Propositions 3 and 4, along with Koebe's 1/4-theorem (12) yield

$$
\begin{aligned}
\frac{|S h(z)|}{\lambda_{\Omega}(z)^{2}} & \leq\left\|S_{f}\right\|_{\Omega}+\frac{2\left|\omega^{*}(z)\right|}{d(z) \lambda_{\Omega}(z)} \sqrt{1+\frac{\|S h\|_{\Omega}}{2}}+\frac{C\left\|\omega^{*}\right\|}{d(z)^{2} \lambda_{\Omega}(z)^{2}}+\frac{3}{2}\left|\omega^{*}(z)\right|^{2} \\
& \leq t+8\left\|\omega^{*}\right\| \sqrt{1+\frac{M}{2}}+16 C\left\|\omega^{*}\right\|+\frac{3}{2}\left\|\omega^{*}\right\|^{2} .
\end{aligned}
$$

Since $\left\|\omega^{*}\right\| \leq 1$ we get that

$$
\|S h\|_{\Omega} \leq t+\hat{C} R_{t}(\Omega)
$$

for some constant $\hat{C}>0$. Hence, $\|S h\|_{\Omega} \rightarrow 0$ as $t \rightarrow 0^{+}$, in view of Lemma 5 . Since $\sigma(\Omega)>0$ by Theorem A, there exists $t_{0}>0$ for which $\|S h\|_{\Omega}<\sigma(\Omega)$, so that $h$ is univalent in $\Omega$. Moreover, $h$ has a quasiconformal extension to $\overline{\mathbf{C}}$ by Theorem A, which we will denote by $\widetilde{h}$.

Using the affine invariance of $\mathcal{F}_{t}(\Omega)$, the above calculation can be repeated for the transform $A_{a} f$, given in (9), for any $a \in \mathbf{D}$. Thus, the analytic part of $A_{a} f$ and, therefore, $h_{a}=h+a g$ is univalent in $\Omega$ for every $a \in \mathbf{D}$. Letting $|a| \rightarrow 1^{-}$and using Hurwitz' theorem we get that $h_{a}$ is univalent in $\Omega$ for every $a \in \mathbf{T}$, since it can not be 
constant due to its normalization $h_{a}^{\prime}(0)=1+a g^{\prime}(0) \neq 0$. We show that $f$ is injective in $\Omega$ by contradiction. Let $z_{1}, z_{2} \in \Omega$ be distinct, and such that $f\left(z_{1}\right)=f\left(z_{2}\right)$. Since $h$ is injective, we have that $h\left(z_{1}\right) \neq h\left(z_{2}\right)$. Setting $\theta=\arg \left(h\left(z_{1}\right)-h\left(z_{2}\right)\right)$ we see that

$$
\mathbf{R} \ni e^{-i \theta}\left(h\left(z_{1}\right)-h\left(z_{2}\right)\right)=e^{-i \theta}\left(\overline{g\left(z_{2}\right)}-\overline{g\left(z_{1}\right)}\right)=e^{i \theta}\left(g\left(z_{2}\right)-g\left(z_{1}\right)\right),
$$

from which we get that $h+e^{2 i \theta} g$ is not injective, a contradiction.

Moreover, $h_{a}$ has a quasiconformal extension $\widetilde{h_{a}}$ to $\overline{\mathbf{C}}$ for every $a \in \mathbf{D}$. In view of Theorem 5.3 in [15, Ch. II, §5.4], for every $a \in \mathbf{T}$ the limit function $\widetilde{h_{a}}$ is either constant, or takes two values, or is quasiconformal. The first two cases are discarded by the normalization at the origin and, therefore, $\widetilde{h_{a}}$ is quasiconformal in $\overline{\mathbf{C}}$ for every $a \in \overline{\mathbf{D}}$. Now we define

$$
\widetilde{f}=\widetilde{h}+\widetilde{\widetilde{g}}
$$

where $\widetilde{h}=\widetilde{h_{0}}$ and $\widetilde{g}=\widetilde{h_{1}}-\widetilde{h_{0}}$. It is clear that $\widetilde{f}$ is a continuous (with respect to the spherical metric) extension of $f$ to $\overline{\mathbf{C}}$. It remains to show that $\widetilde{f}$ is injective in $\overline{\mathbf{C}}$, since the continuity of $\widetilde{f}^{-1}$ may then be obtained by a general result, see Theorem 5.6 in $[17, \S 3-5]$.

We will argue again by contradiction. Let $z_{1}, z_{2} \in \overline{\mathbf{C}}$ be distinct, and such that $\ell=\widetilde{f}\left(z_{1}\right)=\widetilde{f}\left(z_{2}\right) \in \overline{\mathbf{C}}$. Since $\widetilde{h}$ is injective, we have that $\widetilde{h}\left(z_{1}\right) \neq \widetilde{h}\left(z_{2}\right)$. If both $\widetilde{h}\left(z_{1}\right)$ and $\widetilde{h}\left(z_{2}\right)$ are finite then we proceed as in (13), setting $\theta=\arg \left(\widetilde{h}\left(z_{1}\right)-\widetilde{h}\left(z_{2}\right)\right)$ and seeing that $\widetilde{h}+e^{2 i \theta} \widetilde{g}$ is not injective, a contradiction. If one of $\widetilde{h}\left(z_{1}\right)$ and $\widetilde{h}\left(z_{2}\right)$ is finite and the other is infinite, we may assume without loss of generality that $\widetilde{h}\left(z_{1}\right)=\infty$ and $\widetilde{h}\left(z_{2}\right) \neq \infty$. Let $\gamma_{\theta}$ be the pre-image of the ray $\left\{R e^{i \theta}: R \geq 0\right\}, \theta \in[0,2 \pi)$, of the function $\widetilde{h}$. Clearly, $\gamma_{\theta}$ is a simple curve with one endpoint at the origin and the other at $z_{1}$. We write

$$
\widetilde{h}+\widetilde{g}=\overline{\widetilde{f}}+\widetilde{h}-\overline{\widetilde{h}}
$$

and see that for $z \in \gamma_{\theta}$ we have that $\widetilde{h}(z)-\overline{\widetilde{h}}(z)=R e^{i \theta}\left(1-e^{-2 i \theta}\right)$. We distinguish two cases: $\ell$ (the common value of $\widetilde{f}$ at $z_{1}$ and $z_{2}$ ) being finite or infinite. If $\ell \neq \infty$ then

$$
\lim _{\gamma_{\theta} \ni z \rightarrow z_{1}} \tilde{h}(z)+\widetilde{g}(z)= \begin{cases}\bar{\ell}, & \text { if } \theta=0, \pi, \\ \infty, & \text { if } \theta \neq 0, \pi,\end{cases}
$$

which is a contradiction since $\widetilde{h}+\widetilde{g}$ is continuous in $\overline{\mathbf{C}}$. If $\ell=\infty$ we take limit when $z \rightarrow z_{1}$ along $\gamma_{0}$ (so that $\widetilde{h}(z)=R$ ) in order to compute that $(\widetilde{h}+\widetilde{g})\left(z_{1}\right)=\infty$. But, on the other hand, we have that $(\widetilde{h}+\widetilde{g})\left(z_{2}\right)=\infty$, since $\widetilde{h}\left(z_{2}\right) \neq \infty$ and $\widetilde{g}\left(z_{2}\right)=\infty$. This is a contradiction because $\widetilde{h}+\widetilde{g}$ is injective.

\section{Proof of Theorem 3}

Let $\varphi: \mathbf{D} \rightarrow \Omega$ be a Riemann map of $\Omega$ with $\varphi(0)=0$ and consider $\Omega_{r}=\varphi(\{|z|<$ $r\}$ ) for $r<1$. We set $\gamma_{r}=\partial \Omega_{r}$ and note that, since $\Omega$ is a $\widehat{K}$-quasidisk for some $\widehat{K} \geq 1$, it follows (trivially) that $\gamma_{r}$ is a $\widehat{K}$-quasicircle for every $r<1$. We also set $\Gamma_{r}=f\left(\gamma_{r}\right)$ and claim that it is a $K$-quasicircle, with $K \geq 1$ independent of $r$. Once we prove this claim we may then consider $\lambda_{r}$ and $\Lambda_{r}$ to be $\widehat{K}$ - and $K$-quasiconformal reflecions across $\gamma_{r}$ and $\Gamma_{r}$, respectively, and setting

$$
\tilde{f}_{r}(z)= \begin{cases}f(z), & \text { if } z \in \overline{\Omega_{r}}, \\ \Lambda_{r} \circ f \circ \lambda_{r}(z), & \text { if } z \in \overline{\mathbf{C} \backslash \overline{\Omega_{r}},}\end{cases}
$$


we see that this is a $\left(K \frac{1+d}{1-d} \widehat{K}\right)$-quasiconformal mapping in the Riemann sphere. Letting $r \rightarrow 1$, and in view of Theorem 5.3 in [15, Ch. II, §5.4], we obtain the desired quasiconformal extension of $f$ to $\overline{\mathbf{C}}$.

Let $w_{j} \in \Gamma_{r}, j=1,2,3,4$, be distinct points. Our claim that $\Gamma_{r}$ is a $K$-quasicircle, with $K \geq 1$ independent of $r$, will be proved by showing that the cross-ratio of the points $w_{j}$ is bounded by a uniform constant, independent of $r$. Since $f$ is injective in $\Omega$ by Theorem 1 , there exist exactly four points $z_{j} \in \gamma_{r}$ for which $w_{j}=f\left(z_{j}\right)$. For convenience, we will write $h_{j}=h\left(z_{j}\right)$ and $g_{j}=g\left(z_{j}\right)$. We have

$$
w_{i}-w_{j}=h_{i}-h_{j}+\overline{g_{i}}-\overline{g_{j}}=\left(h_{i}-h_{j}\right)\left(1+\mu_{i j} A_{i j}\right),
$$

where $A_{i j}=\frac{g_{i}-g_{j}}{h_{i}-h_{j}}$ and $\mu_{i j}=\frac{\overline{g_{i}}-\overline{g_{j}}}{g_{i}-g_{j}} \in \mathbf{T}$ (we may set $\mu_{i j}=1$ if $g_{i}=g_{j}$ ). We have that

$$
\left|\left(w_{1}, w_{2}, w_{3}, w_{4}\right)\right|=\left|\left(h_{1}, h_{2}, h_{3}, h_{4}\right)\right|\left|\frac{\left(1+\mu_{12} A_{12}\right)\left(1+\mu_{34} A_{34}\right)}{\left(1+\mu_{13} A_{13}\right)\left(1+\mu_{24} A_{24}\right)}\right| .
$$

Since $h(\Omega)$ is a quasidisk (in view of the proof of Theorem 1 ), we have that

$$
\left|\left(h_{1}, h_{2}, h_{3}, h_{4}\right)\right| \leq M
$$

for some absolute constant $M \geq 0$.

We saw in the course of the proof of Theorem 1 that $h_{a}=h+a g$ is univalent in $\Omega$ for every $a \in \overline{\mathbf{D}}$. We will now expand the range of $|a|$ for which this holds.

Let $\delta \in(1,1 / d)$ and consider $1<|a| \leq \delta$. We compute $h_{a}^{\prime}=h^{\prime}(1+a \omega)$ and

$$
\frac{h_{a}^{\prime \prime}}{h_{a}^{\prime}}=\frac{h^{\prime \prime}}{h^{\prime}}+\frac{a \omega^{\prime}}{1+a \omega}
$$

so that

$$
S h_{a}=S h-\frac{a \omega^{\prime}}{1+a \omega} \frac{h^{\prime \prime}}{h^{\prime}}+\frac{a \omega^{\prime \prime}}{1+a \omega}-\frac{3}{2}\left(\frac{a \omega^{\prime}}{1+a \omega}\right)^{2} .
$$

By formula (3) and a straightforward computation we arrive at

$$
S h_{a}=S_{f}+\frac{a+\bar{\omega}}{1+a \omega}\left[\frac{\omega^{\prime \prime}}{1-|\omega|^{2}}-\frac{\omega^{\prime}}{1-|\omega|^{2}} \frac{h^{\prime \prime}}{h^{\prime}}+\frac{3}{2}\left(\frac{\omega^{\prime}}{1-|\omega|^{2}}\right)^{2}\left(\bar{\omega}-\frac{a\left(1-|\omega|^{2}\right)}{1+a \omega}\right)\right] \text {. }
$$

In $\Omega$, we have that

$$
\left|\frac{a+\bar{\omega}}{1+a \omega}\right| \leq \max _{|\zeta|=d}\left|\frac{a+\bar{\zeta}}{1+a \zeta}\right|=\frac{|a|-d}{1-|a| d} \leq \frac{\delta-d}{1-\delta d}=C
$$

where the first of these inequalities follows from the maximum principle. Moreover, we have that

$$
\left|\bar{\omega}-\frac{a\left(1-|\omega|^{2}\right)}{1+a \omega}\right| \leq d+\frac{|a|\left(1-|\omega|^{2}\right)}{1-|a \omega|} \leq d+\frac{|a|\left(1-d^{2}\right)}{1-|a| d} \leq d+\frac{\delta\left(1-d^{2}\right)}{1-\delta d}=C^{\prime} .
$$

Hence, we get that

$$
\left|S h_{a}\right| \leq\left|S_{f}\right|+C\left[\frac{\left|\omega^{\prime \prime}\right|}{1-|\omega|^{2}}+\frac{\left|\omega^{\prime}\right|}{1-|\omega|^{2}}\left|\frac{h^{\prime \prime}}{h^{\prime}}\right|+\frac{3 C^{\prime}}{2}\left(\frac{\left|\omega^{\prime}\right|}{1-|\omega|^{2}}\right)^{2}\right] .
$$

We assume that $f \in \mathcal{F}_{t}(\Omega)$, for $t \geq 0$, and, working as in the proof of Theorem 1 , we use Propositions 3, 4 and Koebe's 1/4-theorem (12) in order to obtain

$$
\left\|S h_{a}\right\|_{\Omega} \leq t+\hat{C} R_{t}(\Omega)
$$

for some constant $\hat{C}>0$. Choosing $t_{0}>0$ so that $t_{0}+\hat{C} R_{t_{0}}(\Omega)=\sigma(\Omega)$ we conclude that $h_{a}$ is univalent. 
We fix $\alpha \in \Omega$ and consider the generalized dilatation

$$
\psi_{\alpha}(z)= \begin{cases}\frac{g(z)-g(\alpha)}{h(z)-h(\alpha)}, & \text { if } z \in \Omega \backslash\{\alpha\} \\ \omega(\alpha), & \text { if } z=\alpha .\end{cases}
$$

Since $h$ is injective in $\Omega$ it is clear that $\psi_{\alpha}$ is holomorphic in $\Omega$. We claim that

$$
S=\max _{z \in \overline{\Omega_{r}}}\left|\psi_{\alpha}(z)\right| \leq \frac{1}{\delta}
$$

Note that $|\omega(\alpha)| \leq d<1 / \delta$ so that if, otherwise, $S>1 / \delta$, then there would exist a point $z_{0} \in \Omega \backslash\{\alpha\}$ for which $\psi_{\alpha}\left(z_{0}\right)=e^{i \theta} / \delta$, for some $\theta \in \mathbf{R}$. This shows that the values of the function $h-e^{-i \theta} \delta g$ at the points $\alpha$ and $z_{0}$ would coincide, which is a contradiction since this is a univalent function.

Hence we may bound the terms in (14) as $\left|A_{i j}\right| \leq 1 / \delta$ in order to obtain

$$
\left|\left(w_{1}, w_{2}, w_{3}, w_{4}\right)\right| \leq M \frac{\left(1+\left|A_{12}\right|\right)\left(1+\left|A_{34}\right|\right)}{\left(1-\left|A_{13}\right|\right)\left(1-\left|A_{24}\right|\right)} \leq M\left(\frac{1+1 / \delta}{1-1 / \delta}\right)^{2},
$$

with which we finish the proof.

\section{Finitely connected domains}

Let $\Omega$ be a domain in $\overline{\mathbf{C}}$. A collection $\mathfrak{D}$ of domains $D \subset \Omega$ is called a quasiconformal decomposition of $\Omega$ if each $D$ is a quasidisk and any two points $z_{1}, z_{2} \in \Omega$ lie in the closure of some $D \in \mathfrak{D}$. This definition along with the following covering lemma were given by Osgood in [18].

Lemma C. [18] If $\Omega$ is a finitely connected domain and each component of $\partial \Omega$ is either a point or a quasicircle then $\Omega$ is quasiconformally decomposable.

The proof in [18] provides an explicit finite decomposition.

Proof of Therorem 2. We prove the direction (i) $\Rightarrow$ (iii). The domain $\Omega$ is quasiconformally decomposable by a collection $\mathfrak{D}$ in view of Lemma C. By Theorem 1 , each of the quasidisks $D$ in $\mathfrak{D}$ has positive harmonic inner radius. Let

$$
0<c \leq \min _{D \in \mathfrak{D}} \sigma_{H}(D)
$$

and consider $f$ to be a harmonic mapping in $\Omega$ that satisfies $\left\|S_{f}\right\|_{\Omega} \leq c$. If $f\left(z_{1}\right)=$ $f\left(z_{2}\right)$ for two distinct points $z_{1}, z_{2}$ in $\Omega$ then $z_{1}, z_{2} \in \bar{D}$, for some quasidisk $D$ from the collection $\mathfrak{D}$. The domain monotonicity for the hyperbolic metric shows that $\lambda_{D}(z) \geq \lambda_{\Omega}(z)$ for all $z \in D$ and, therefore, that

$$
\left\|S_{f}\right\|_{D} \leq\left\|S_{f}\right\|_{\Omega} \leq c .
$$

But now the homeomorphic extension of Theorem 1 shows that if $c$ is sufficiently small then $f$ is injective up to the boundary of $D$, a contradiction.

We note that if we strengthen the definition of quasiconformal decomposition so that any two points $z_{1}, z_{2} \in \Omega$ lie in some quasidisk $D$ (not its closure) from a collection $\mathfrak{D}$, then the construction in [18] can be modified so that Lemma $\mathrm{C}$ still holds. Had we followed this line of reasoning then we would not need the homeomorphic extension of Theorem 1, but only the univalence criterion from its statement.

Acknowledgements. I would like to thank Professor Martin Chuaqui for his insightful comments in our many discussions. 


\section{References}

[1] Ahlfors, L.: Quasiconformal reflections. - Acta Math. 109, 1963, 291-301.

[2] Beardon, A. F., and F. W. Gehring: Schwarzian derivatives, the Poincaré metric and the kernel function. - Comment. Math. Helv. 55:1, 1980, 50-64.

[3] Beardon, A., and D. Minda: The hyperbolic metric and geometric function theory. - In: Quasiconformal mappings and their applications, Narosa, New Delhi, 2007, 9-56.

[4] Chunqui, M., P. Duren, and B. Osgood: The Schwarzian derivative for harmonic mappings. - J. Anal. Math. 91, 2003, 329-351.

[5] Chunqui, M., R. Hernández, and M. J. Martín: Affine and linear invariant families of harmonic mappings. - Math. Ann. 367:3-4, 2017, 1099-1122.

[6] Duren, P. L.: Harmonic mappings in the plane. - Cambridge Univ. Press, Cambridge, 2004.

[7] Gehring, F. W.: Univalent functions and the Schwarzian derivative. - Comment. Math. Helv. $52: 4,1977,561-572$.

[8] Gehring, F. W., and K. Hag: The ubiquitous quasidisk. - Amer. Math. Soc., Providence, RI, 2012.

[9] Ghatage, P., and D. Zheng: Hyperbolic derivatives and generalized Schwarz-Pick estimates. - Proc. Amer. Math. Soc. 132:11, 2004, 3309-3318.

[10] Hernández, R., and M. J. Martín: Quasiconformal extension of harmonic mappings in the plane. - Ann. Acad. Sci. Fenn. Math. 38:2, 2013, 617-630.

[11] Hernández, R., and M. J. Martín: Pre-Schwarzian and Schwarzian derivatives of harmonic mappings. - J. Geom. Anal. 25:1, 2015, 64-91.

[12] Hernández, R., and M. J. Martín: Criteria for univalence and quasiconformal extension of harmonic mappings in terms of the Schwarzian derivative. - Arch. Math. (Basel) 104:1, 2015, 53-59.

[13] Kühnau, R.: Möglichst konforme Spiegelung an einer Jordankurve. - Jahresber. Deutsch. Math.-Verein. 90:2, 1988, 90-109.

[14] Lehto, O.: Univalent functions and Teichmüller spaces. - Grad. Texts in Math. 109, SpringerVerlag, New York, 1987.

[15] Lehto, O., and K. I. Virtanen: Quasiconformal mappings in the plane. Second edition. Springer-Verlag, New York-Heidelberg, 1973.

[16] Martio, O., and J. Sarvas: Injectivity theorems in plane and space. - Ann. Acad. Sci. Fenn. Ser. A I Math. 4:2, 1979, 383-401.

[17] Munkres, J. R.: Topology, a first course. - Prentice-Hall, Inc., Englewood Cliffs, N.J., 1975.

[18] Osgood, B.: Univalence criteria in multiply-connected domains. - Trans. Amer. Math. Soc. 260:2, 1980, 459-473.

[19] Pommerenke, Ch.: Linear-invariante Familien analytischer Funktionen I. - Math. Ann. 155:2, $1964,108-154$.

Received 1 October 2020 • Accepted 18 June 2021 